\title{
Probing pre-formed alpha particles in the ground state of nuclei.
}

\author{
J.A. Scarpaci ${ }^{1}$, M. Fallot ${ }^{1, *}$, D. Lacroix ${ }^{2}$, M. Assié ${ }^{1}$, L. Lefebvre ${ }^{1}$, N. Frascaria ${ }^{1}$, D. Beaumel ${ }^{1}$, C. Bhar ${ }^{1}$, Y. \\ Blumenfeld $^{1}$, A. Chbihi ${ }^{2}$, Ph. Chomaz ${ }^{2, \dagger}$, P. Desesquelles ${ }^{1, * *}$, J. Frankland ${ }^{2}$, H. Idbarkach ${ }^{1}$, E. Khan ${ }^{1}$, J.L. Laville ${ }^{2}$, \\ E. Plagnol ${ }^{1, * * *}$, E.C. Pollacco ${ }^{3}$, P. Roussel-Chomaz ${ }^{2}$, J.C. Roynette ${ }^{1}$, A. Shrivastava ${ }^{1, * * * *}$, T. Zerguerras ${ }^{1}$ \\ ${ }^{1}$ Institut de Physique Nucléaire, CNRS/IN2P3, Université Paris-Sud 11, F-91406 Orsay, France \\ ${ }^{2}$ GANIL, CEA/DSM - CNRS/IN2P3, Bd Henri Becquerel, BP 55027, F-14076 Caen Cedex 5, France and \\ ${ }^{3}$ CEA-Saclay, DSM/IRFU SPhN, F-91191 Gif sur Yvette Cedex, France
}

(Dated: November 19, 2018)

\begin{abstract}
In this Letter, we report on alpha particle emission through the nuclear break-up in the reaction ${ }^{40} \mathrm{Ca}$ on a ${ }^{40} \mathrm{Ca}$ target at $50 \mathrm{~A} \mathrm{MeV}$. It is observed that, similarly to nucleons, alpha particles can be emitted to the continuum with very specific angular distribution during the reaction. The alpha particle properties can be understood as resulting from an alpha cluster in the daughter nucleus that is perturbed by the short range nuclear attraction of the collision partner and emitted. A time-dependent theory that describe the alpha particle wave-function evolution is able to reproduce qualitatively the observed angular distribution. This mechanism offers new possibilities to study alpha particle properties in the nuclear medium.
\end{abstract}

\section{PACS:21.60.Gx \\ Keywords:Alpha clustering}

Nuclei are complex self-bound systems formed of nucleons. Conjointly to a mean-field picture where nucleons can be regarded as independent particles, few nucleons might self-organize into compact objects, called clusters, inside the nucleus. The understanding of clustering in the nuclear medium is a central issue and has been the subject to extensive research. Clustering in $N=Z$ nuclei has a long standing history (see discussion in [1]) and recent AMD calculation are studying the $\alpha+{ }^{36} \mathrm{Ar}$ configuration of the ${ }^{40} \mathrm{Ca}$ nucleus [2] for which experimental results are presented here. The states based on $\alpha$ particles have also been observed in $\left(\mathrm{d},{ }^{6} \mathrm{Li}\right)$ experiment on ${ }^{40} \mathrm{Ca}[3]$, however they are usually not so much found in the ground states (GS) but rather observed as excited states close to the decay thresholds into clusters, as suggested by Ikeda [4]. In particular, the Hoyle state [5], i.e., the $0_{2}^{+}$state at $7.65 \mathrm{MeV}$ in ${ }^{12} \mathrm{C}$ which was interpreted as an alpha-particle condensate [6], and other similar states in heavier n $\alpha$ nuclei, have attracted much renewed attention (see, e.g., [7]). Other experiments have observed the emission of alpha particles but with different mechanisms such as the decay of giant resonances [8] or the emission from the neck [9].

In this Letter we report on sudden $\alpha$ particle emission resulting of the nuclear break-up, also called the "Towing mode", of $\alpha$ cluster present in the ground state of ${ }^{40} \mathrm{Ca}$. This mechanism, first observed with neutrons and protons in the inelastic scattering channels with stable beams [10] is an emission to the continuum due to the nuclear potential of the passing by nucleus. The time scale is much shorter here than for a regular evaporation as it is related to passing time of the projectile of the order of $10^{-22} \mathrm{~s}$. It was understood with a dedicated Time Dependent Schrödinger Equation (TDSE) [1] technique as follows. As the emitter nucleus passes close to the collision partner, the short-range nuclear potential of the latter attracts the least bound nucleons leading to the emission of particles at mid-rapidity. We have shown an excellent reproduction of the experimental properties with the model calculation and we have demonstrated that this mechanism has several interesting aspects. The characteristics of the emission (angular and energy distributions) strongly depend on the initial quantum properties of the towed particle (angular momentum, extension of the wave-function and binding energy). These properties promote the "Towing mode" as a tool of choice to infer spectroscopic information of nucleons in nuclei as shown in ref. [12 14 for ${ }^{11} \mathrm{Be}$ and ${ }^{6} \mathrm{He}$.

In this present experiment, it has been observed not only the target break-up of one proton [15], but also of $\alpha$ particle [8] as we will present in the following, indicating the formation of $\alpha$ cluster in the ${ }^{40} \mathrm{Ca}$ nucleus. The $\alpha$ cluster is emitted to the continuum due to the perturbation of the other nucleus with specific angular and energetic properties. A dedicated model describing the evolution of the $\alpha$ particle wave-function initially in the daughter nucleus for grazing impact parameters is able to describe qualitatively the observed behavior.

The experiment was performed at the GANIL facility, by bombarding a self-supported $0.2 \mathrm{mg} / \mathrm{cm}^{2}$ natural Ca target with a $50 \mathrm{~A} \mathrm{MeV}{ }^{40} \mathrm{Ca}$ beam. The ejectiles were identified in the focal plane of the SPEG spectrometer [16], in coincidence with the light charged particles detected in $240 \mathrm{CsI}(\mathrm{Tl})$ detectors of the INDRA $4 \pi$ array [17] covering an angular range from $14^{\circ}$ to $176^{\circ}$ with respect to the beam direction. The projectiles were measured between $\theta_{\text {lab }}=0.5^{\circ}$ and $5^{\circ}$ using the SPEG spectrometer with its standard detection system. Stripped cathode drift chambers were used for the position measurement in the focal plane [18]. Combined with the very thin target, they allowed for an excitation energy resolution of $350 \mathrm{keV}$, i.e., $\Delta p / p=9 \times 10^{-5}$. The ejectiles were unambiguously identified in charge and mass through energy and time of flight measurements and in this letter we will concentrate on the inelastic channel only where the ejectiles are of the same nature as the ${ }^{40} \mathrm{Ca}$ projectile. 


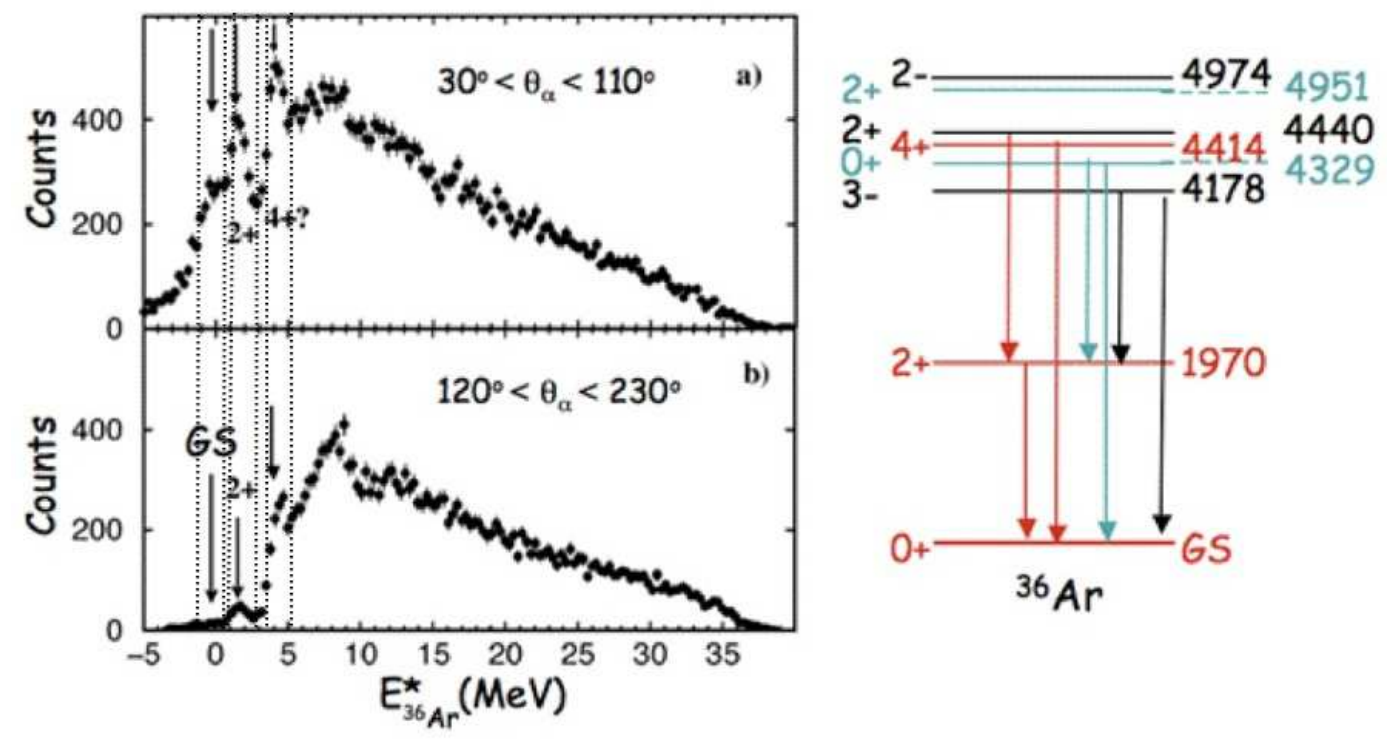

FIG. 1: (Color online) Missing energy spectrum of ${ }^{36} \mathrm{Ar}$ in the reaction ${ }^{40} \mathrm{Ca}\left({ }^{40} \mathrm{Ca},{ }^{40} \mathrm{Ca}+\alpha\right)$ for an excitation energy range between 20 and $50 \mathrm{MeV}$. Fig. (a): $\alpha$ particles are detected between 30 and 110 degrees. (b): $\alpha$ particles are detected between 130 and 220 degrees in the laboratory system, in the backward direction compared to the beam. The arrows show the GS, the $2^{+}$state and a state around 4.4 MeV. The dashed lines are the upper and lower limits of the gates used to extract the angular distributions presented in Fig2 Lower and higher values for the gates were as follows: from -1.1 to $0.7 \mathrm{MeV}$ to select most of the GS ; from 1.1 to $2.9 \mathrm{MeV}$ for the $2^{+}$state and from 3.7 to 5.1 for state around $4 \mathrm{MeV}$. The Right panel shows the level scheme of known states up to $4.974 \mathrm{MeV}$ excitation energy in ${ }^{36} \mathrm{Ar}$ [20].

The identification of the particles was obtained by analyzing the fast and slow components of the CsI scintillator light, and was fully reliable above particle kinetic energy of $4 \mathrm{MeV}$. We have therefore set a $4 \mathrm{MeV}$ software energy threshold for the $\alpha$ particles. The light particle energy calibration was performed using the particle decay towards the GS of the daughter nucleus [15, 19]. The obtained energy resolution for $\alpha$ particles is $1.1 \mathrm{MeV}$ hence a $1.15 \mathrm{MeV}$ energy resolution on the reconstructed missing energy (see below).

We performed a measurement of the missing energy calculated as follows: $E_{\text {miss }}=E_{\mathrm{Ca}}^{*}-E_{\alpha}^{\mathrm{CM}}-E_{\text {recoil }}\left({ }^{36} \mathrm{Ar}\right)$ where $E_{\mathrm{Ca}}^{*}$ is the initial excitation energy in ${ }^{40} \mathrm{Ca}$ obtained by the measurement of the inelastically scattered projectile detected after the SPEG spectrometer, $E_{\alpha}^{\mathrm{CM}}$ the $\alpha$ particle energy in the center of mass frame of the recoiling ${ }^{40} \mathrm{Ca}$ target, and $E_{\text {recoil }}$ is the recoil energy of target-like ${ }^{36} \mathrm{Ar}$. The remaining excitation energy of ${ }^{36} \mathrm{Ar}$ is then $\mathrm{E}_{36}^{*} A r=\mathrm{E}_{\text {miss }}-\mathrm{Q}_{\alpha}$ and is plotted in Fig 1. We show the final states of ${ }^{36} \mathrm{Ar}$ after an $\alpha$ particle was removed from the target and detected in two different angular regions (for an apparent excitation energy range between 20 and $50 \mathrm{MeV}$ ). Spectra (a) and (b) correspond to forward and backward emission respectively. Region (b) is selecting the evaporation of an excited ${ }^{40} \mathrm{Ca}$ target that shall emit isotropically. This statistical decay is then also present in Fig!1(a) with on top a strong feeding of several low energy states of ${ }^{36} \mathrm{Ar}$. On spectrum (a), we clearly recognize the $0^{+}$GS, the first $2^{+}$state at $1.97 \mathrm{MeV}$ and a second excited state around $4.4 \mathrm{MeV}$ which could not be separated from the surrounding states (see right panel of Fig (10) but could possibly be assigned to the $4^{+}$state of the ${ }^{36} \mathrm{Ar}$. On spectrum (b), these states are much less populated. The difference observed between the forward and backward $\alpha$ particle emission is very similar to the anisotropy in nucleon emission measured previously in reaction ${ }^{58} \mathrm{Ni}\left({ }^{40} \mathrm{Ar},{ }^{40} \mathrm{Ar}+\mathrm{p}\right.$ or $\left.\mathrm{n}\right){ }^{57} \mathrm{X}$ (see Ref.[10]) which is a clear signature of nuclear break-up, called sometime "Towing mode" where the target after its break-up is left in its GS or with very small excitation energy. Because of this sudden nuclear break-up, the $\alpha$ particle is expelled from a cold nucleus, thus the idea of a pre-formation in the GS of this nucleus.

This first evidence of a similar phenomenon with $\alpha$ particles motivates the precise understanding of this observation in order to provide a new tool for the study of $\alpha$ particle properties in the nuclear medium. Extrapolating results obtained with nucleons, we do expect that the properties of the emitted particles depend significantly on the initial $\alpha$ particle properties in the emitter (binding energy, quantum numbers, wave-function extension). Here, we will follow exactly the same strategy as for single nucleon emission and question if the observed anisotropy in $\alpha$ angular distribution can be understood as the nuclear break-up of a preformed $\alpha$ particle wave-function.

The mechanism responsible for anisotropic distribution 


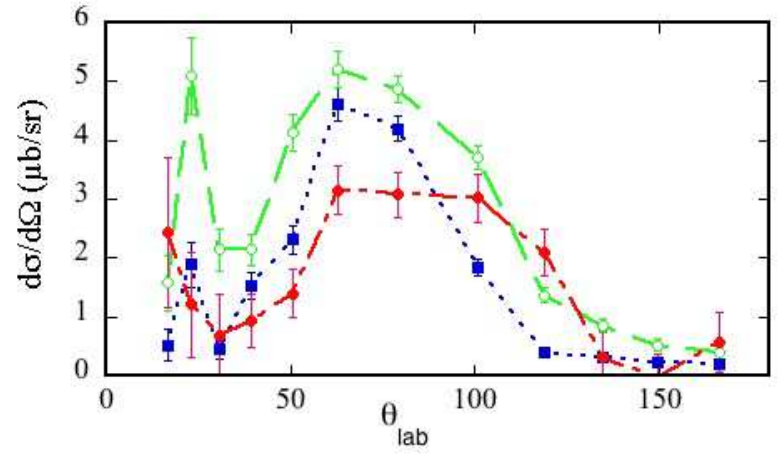

FIG. 2: (Color online) Angular cross-sections of $\alpha$ particles when the three first states around $E_{36}^{*} \mathrm{Ar} \simeq 0$ (blue dotted line), 2 (green dashed line) and $4 \mathrm{MeV}$ (red dot-dashed line) in figure 1 are fed respectively. For the later state, a subtraction was made with the distribution obtained for the 8 to $9 \mathrm{MeV}$ region.

of $\alpha$ particles seems to feed different states of a band corresponding to a deformed ${ }^{36} \mathrm{Ar}$ nucleus with a $\beta$ of 0.256 [21] (see Fig[1 right). The feeding of several states of the ${ }^{36} \mathrm{Ar}$ can eventually be understood as follows. Assuming that the $0^{+}$GS of the ${ }^{40} \mathrm{Ca}$ presents different configurations corresponding to an $\alpha$ particle wave-function coupled to an ${ }^{36} \mathrm{Ar}$ core, eventually excited, we write a configuration as $\left(\left|\varphi_{\alpha}\right\rangle \otimes\left|\left({ }^{36} \mathrm{Ar}\right)^{*}\right\rangle\right)_{n}$ where $n$ labels the configuration while $\varphi_{\alpha}$ and $\left|\left({ }^{36} \mathrm{Ar}\right)^{*}\right\rangle$ denote respectively a specific $\alpha$ particle wave-function and a specific state of the ${ }^{36} \mathrm{Ar}$. As the $\alpha$ particle is emitted, the ${ }^{36} \mathrm{Ar}$ is left in the specific configuration $\left|\left({ }^{36} \mathrm{Ar}\right)^{*}\right\rangle$ and can eventually decay through $\gamma$ emission. Assuming that the $\alpha$ configurations present in the ${ }^{40} \mathrm{Ca}$ GS do not interfere with each others, the feeding of different states can directly be assigned to the independent contributions of each configuration. A complete understanding of the feeding process and/or the extraction of $\alpha$ particle spectroscopic factors would require very good $\alpha$ particle energy resolution as well as $\gamma$ coincidence. Due to absence of gamma detection and insufficient energy resolution $(1.15 \mathrm{MeV})$ of the missing energy spectrum (Fig 1), separation between the GS and the first $2^{+}$state, and the states around $4 \mathrm{MeV}$ in ${ }^{36} \mathrm{Ar}$ could not be precisely achieved. However, in spite of these limitations, the angular cross-section of the emitted $\alpha$ particles could be extracted. Here, the compatibility of the scenario described above will be tested qualitatively on the angular distributions of the $\alpha$ particles associated with a particular state in the ${ }^{36} \mathrm{Ar}$ against the experimental observation.

In order to isolate and characterize the contribution of a given configuration, the experimental $\alpha$ particle angular distributions were extracted by setting gates around the GS, the first $2^{+}$state and the state about $4 \mathrm{MeV}$, bearing in mind that this selection allows for some contribution from one state to the others. These gates are presented on the left spectra of Fig 1 (vertical dashed lines) and the corresponding distributions are displayed in Fig 2, For the state around $4 \mathrm{MeV}$, as it has some contribution from evaporation as seeing in Fig 1 b), a subtraction was made of the 8 to $9 \mathrm{MeV}$ region which only decays through evaporation and that shows a rather flat contribution (not shown here). A clear sensitivity of the angular distribution to the final state is observed in this figure.

To test if these distributions could be understood as the final product of an $\alpha$ particle initially in the ${ }^{40} \mathrm{Ca}$ and emitted to the continuum as the projectile passes by, our TDSE technique was applied to the emission of alphas. In this model, an $\alpha$ particle wave-function, denoted by $\varphi_{\alpha}$ is first initialized in a spherical symmetric potential describing the core-alpha interaction. The potential of ref. [22], which has been optimized for nuclei in the same mass region to reproduce nuclear structure spectra, $B\left(E_{2}\right)$ transition strength, $\alpha$ decay widths and elastic scattering cross-sections, is used here. Its nuclear part is given by

$$
V(r)=V_{0}\left\{\frac{\alpha}{\left(1+e^{\left(r-R_{0}\right) / a_{0}}\right)}+\frac{1-\alpha}{\left(1+e^{\left(r-R_{0}\right) / a_{0}}\right)^{3}}\right\}
$$

where the parameters are taken as $V_{0}=-175.7 \mathrm{MeV}$, $a_{0}=0.73 \mathrm{fm}, \alpha=0.3$, and $R_{0}=4.33 \mathrm{fm}$ and is complemented by a Coulomb repulsive potential. As stressed in ref. [22], to approximately account for the Pauli blocking effect coming from the presence of nucleons in the core, only levels with $2 n+L \geq 12$, with $n$ and $L$ being respectively the radial and angular quantum numbers, are considered. For ${ }^{40} \mathrm{Ca}$, the first states that the $\alpha$ particle might occupy are the $6 \mathrm{~s}, 5 \mathrm{~d}$ and $4 \mathrm{~g}$ and have respective binding energies of 7.0, 6.4 and $5.5 \mathrm{MeV}$.

Starting from one of this $\alpha$ particle wave-function, the corresponding nuclear break-up has been studied by solving the Schrödinger equation:

$$
i \hbar \frac{d}{d t} \varphi_{\alpha}(\mathbf{r}, t)=\left\{\frac{\mathbf{p}^{2}}{2 m_{\alpha}}+V_{\alpha \mathrm{Ar}}\left(\mathbf{r}-\mathbf{R}_{T}(t)\right)+V_{\alpha \mathrm{Ca}}\left(\mathbf{r}-\mathbf{R}_{P}(t)\right)\right\} \varphi_{\alpha}(\mathbf{r}, t)
$$




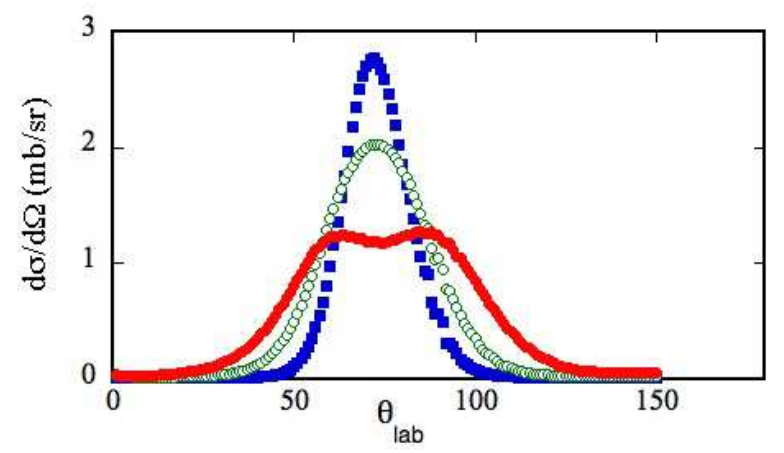

FIG. 3: (Color online) Calculated angular distributions for $\alpha$ particle initially in an 6s (blue squares), 5d (green open circles) and $4 \mathrm{~g}$ (red dots) states in ${ }^{40} \mathrm{Ca}$ and performed with the TDSE calculation.

tials respectively (both taken identical to (10). The target and projectile center of mass evolution, $\mathbf{R}_{T}(t)$ and $\mathbf{R}_{P}(t)$ are chosen to describe a Coulomb trajectory for the passing ${ }^{40} \mathrm{Ca}$ projectile. The TDSE is solved on a 3D mesh of size 259 in $x$ and $y$ ( $y$ being the direction of the beam) and 199 in $z$ with a r-step and time step of $0.2 \mathrm{fm}$ and $1.22 \mathrm{fm} / \mathrm{c}$ respectively.

Similarly to the nucleon case, part of the wave-function that was initially bound by the core is emitted to the continuum. From the wave-function in momentum space, energetic and angular properties of emitted $\alpha$ can be calculated. The final angular distributions for an $\alpha$ particle wave function initially in the $6 \mathrm{~s}, 5 \mathrm{~d}$ and $4 \mathrm{~g}$ states are shown in Fig 3 for an impact parameter between 9.5 and $11.5 \mathrm{fm}$.

These calculations can be compared to the experimental angular distributions that leave the ${ }^{36} \mathrm{Ar}$ nucleus in its GS and the two observed excited states presented in Fig2 Although core+alpha potential used in the calculation is very schematic, it is quite amazing to see the resemblance as for the widths between the three TDSE calculations of the $6 \mathrm{~s}, 5 \mathrm{~d}$ and $4 \mathrm{~g}$ wave functions and the experimental data gated respectively on the three peaks at $E_{36 \mathrm{Ar}}^{*} \simeq 0,2$ and $4 \mathrm{MeV}$ shown in Fig 2 In particular, no adjustment was made to the nuclear potential nor was the possible deformation of the nucleus in which the $\alpha$ particle sits included. In addition, as stressed above, due to $\alpha$ particle energy resolution, it is difficult to separate the different states and some sizable contribution of the neighboring states could be present in the angular distribution extracted for a specific state.

Similarly to experimental data, one could obtain absolute cross sections from the calculations and deduce spectroscopic factor (SF) by comparing theoretical and experimental results. However a precise estimate of SF requires a perfect knowledge of the model parameters and specially of the projectile potential which is beyond the purpose of this letter.

Nevertheless, these findings are very encouraging: (i) they confirm the "Towing mode" scenario for the anisotropic emission of $\alpha$ particle recently observed (ii) it shows that experimental observation can be described assuming a preformed $\alpha$ particle wave-function in the GS of ${ }^{40} \mathrm{Ca}$. (iii) A qualitative description of the experiment is achieved by supposing different contributions coming from different $l$ value of the outgoing $\alpha$ particle.

Experimental evidence was shown for the emission of $\alpha$ particles during the break-up of a ${ }^{40} \mathrm{Ca}$ target. A first comparison was made with a TDSE calculation assuming a preformed $\alpha$ particle in the nucleus and the general behavior of the measured distribution was reproduced. This finding opens new perspectives for the study of preformed $\alpha$ clusters in the nuclear medium. Similarly to the nucleon case, $\alpha$ particles emitted through the nuclear break-up channel have properties that depends sensibly on the initial wave-function (see for instance Fig. 3 for the quantum number dependence). Following the same strategy as in the nucleon case, one might with dedicated experiments, and improved calculations, be able to access pre-formed $\alpha$ particle wave-function properties in the ground state of the nuclei.

$\dagger$ Present address: CEA / Irfu - Centre de Saclay, F91191 Gif sur Yvette Cedex, France

*Present address: SUBATECH (CNRS/IN2P3 - Univ. of Nantes - EMN) F-44307 Nantes Cedex 3, France

**Present address: CSNSM F-91405 Orsay, France

***Present address: APC UMR7164 Université Paris VII F-75012, France

****Present address: Bhabha Atomic Research Center, Mumbai, India
[1] W. von Oertzen, M. Freer, Y. Kanada-Enyo, Phys. Rep. 432, 43 (2006) and references therein.

[2] Y. Taniguchi, M. Kimura, Y. Kanada-Enyo and H. Horiuchi, Phys. Rev. C46 (2007) 044317.

[3] K. Umeda, et al., Nucl. Phys. A429, 88 (1984).

[4] K. Ikeda, T. Marumori, R. Tamagaki, and H. Tanaka, Supplement of the Progress of Theoretical Physics 52, 1 (1972).

[5] M. Chernykh et al., Phys. Rev. Lett. 98, 03250 (2207)

[6] A. Tohsaki et al., Phys. Rev. Lett. 87, 192501 (2001).

[7] P. Schuck et al., Prog. Part. Nucl. Phys. 59, 285 (2007).
[8] M. Fallot.et al., Phys. Lett. B613 (2005) 128

[9] S. Hudan et al., Phys. Rev. C70, 031601 (2004).

[10] J.A. Scarpaci et al., Phys. Lett. B428 (1998) 241.

[11] D. Lacroix, J.A. Scarpaci and Ph. Chomaz, Nucl.Phys. A658 (1999) 273.

[12] V. Lima et al, Nucl. Phys. A795 (2007) 1.

[13] M. Assié et al., Eur. Phys. J. A42, 441 (2009).

[14] M. Assié and D. Lacroix, Phys. Rev. Lett. 102, 202501 (2009).

[15] M. Fallot, Ph.D. Thesis, IPNO 2002 T-02-05, web address: http://tel.ccsd.cnrs.fr/. 
[16] L. Bianchi, et al., Nucl. Inst. Meth. A276 (1989) 509.

[17] J. Pouthas, et al., Nucl. Inst. Meth. A357 (1995) 418.

[18] A. Drouart, Ph.D. Thesis, CEA Saclay, 2000.

[19] J.A. Scarpaci et al., Phys. Rev. C 56 (1997) 3187.

[20] http://www.nndc.bnl.gov/
[21] S. Raman, C.W. Nestor, JR., and P. Tikkanen, Atomic Data and Nuclear Data Tables 78, 1128 (2001)

[22] B. Buck, J. C. Johnston, A. C. Merchant, and S. M. Perez, Phys. Rev. C52, 1840 (1995). 